\title{
Quantum size effect on the phonon-induced Zeeman splitting in a GaAs quantum dot with Gaussian and parabolic confining potentials
}

\author{
Soma Mukhopadhyaya ${ }^{\mathrm{a}, 1}$, Bahadir Boyacioglu ${ }^{\mathrm{b}}$, Mesude Saglam ${ }^{\mathrm{c}}$, Ashok Chatterjee ${ }^{\mathrm{a}, *, 2}$ \\ ${ }^{a}$ Department of Physics, Bilkent University, 06800 Bilkent, Ankara, Turkey \\ ${ }^{\mathrm{b}}$ Vocational School of Health, Ankara University, 06290 Kecioren, Ankara, Turkey \\ ${ }^{\mathrm{c}}$ Department of Physics, Faculty of Sciences, Ankara University, 06100 Tandogan, Ankara, Turkey
}

Received 30 November 2007; accepted 13 December 2007

Available online 9 January 2008

\begin{abstract}
The Zeeman splitting of the ground and the first excited level of a Gaussian GaAs quantum dot is studied in the presence of electron-longitudinal-optical (LO)-phonon interaction incorporating the spin of the electron and is compared with the case of a parabolic dot. It is shown that the Zeeman splitting is suppressed because of the polaronic interaction and becomes strongly size dependent, but the parabolic confinement overestimates this Zeeman suppression. It is also shown that although the energy levels are split because of the spin-field interaction, the cyclotron frequencies and the Zeeman lines are independent of the electron spin in the dipole transition.
\end{abstract}

(C) 2008 Elsevier B.V. All rights reserved.

PACS: 73.20.Dx; 73.20.Mf; 73.40.Kp

Keywords: Gallium arsenide; Quantum dots; Zeeman splitting; Electron-phonon interaction

The subject of quantum dots has remained in the focus of attention for the last three decades or so for both academic and practical reasons [1]. In this context, semiconductor quantum dot structures have drawn particular attention for their potential applications in nano-semiconducting and optoelectronic devices like single electron transistors, quantum dot lasers, ultra-fast computers and so on. Several early experiments [2], and subsequent theoretical investigations [3] have revealed that Kohn's theorem [4] or more precisely a generalization of it is valid in a quantum dot which suggested that the confining potential would be parabolic in such a system. Consequently, a large number of theoretical investigations have been carried out in this area in the last two decades taking the confinement potential as parabolic

\footnotetext{
${ }^{*}$ Corresponding author. Tel.: + 903122902673 ; fax: + 903122664779 .

E-mail addresses: saglam@science.ankara.edu.tr (M. Saglam), acsp@uohyd.ernet.in (A. Chatterjee).

${ }^{1}$ On leave from Shadan Institute of P.G. Studies, Khairatabad, Hyderabad, India.

${ }^{2}$ On leave from School of Physics, University of Hyderabad, Hyderabad, India.
}

[5]. Since most of the quantum dot structures available today are made of polar semiconductors, and the electron-phonon interaction energy scale is almost comparable to the other energy scales of the problem, there have also been several investigations on the polaronic effects in parabolic semiconductor quantum dots and a good deal of literature [6] has already piled up on this subject.

However, in recent years a few groups [7] have observed that their experimental results do not support the validity of the generalized Kohn theorem in quantum dots. Rather, their observations suggest that the confining potential is non-parabolic and should be in the form of a finite well. This has provided a renewed impetus in the research of the electronic energy spectra and the associated phenomena in quantum dots. Using a three-dimensional (3D) spherical rectangular potential well of finite depth, Szafran et al. [8] and Bednarek et al. [9] have been able to successfully describe, albeit qualitatively, the charging of quantum dots. Subsequently, Szafran et al. [10] have quantitatively explained the capacitance-spectroscopy data for selfassembled quantum dots simulating the confining potential 
by a 3D cylindrical potential well. Recently, Adamowsky et al. [11] have proposed an attractive Gaussian confining potential for the investigation of the properties of excess electrons in quantum dots. This potential has a finite depth and in the neighbourhood of the dot centre would behave like a parabolic potential and would also approximately satisfy the generalized Kohn theorem. Furthermore, in contrast to the rectangular potential well, it is continuous at the dot boundaries and this makes it easier to handle mathematically. Also, it has a central minimum as required for a physical potential and the force experienced by the particles within this potential well is non-zero which is also a desirable feature. The other advantages with the Gaussian confining potential vis-a-vis a parabolic potential are that the former allows for, in addition to the excitations, also the ionization (charging) and tunnelling processes. We would like to mention in passing that the Gaussian potential has been used in various branches of physics and has been solved approximately for a single particle problem by several authors [12].

As already mentioned, a large number of investigations have been carried out to study the polaronic effects in parabolic quantum dots (PQDs) and in quantum dots with square and spherical well confining potentials [6], but there has not been much investigation, to our knowledge, with a Gaussian confinement. Since, it has been recently shown [11] that the parabolic potential is only a poor approximation of the more realistic Gaussian potential, it would indeed be important to study the polaronic effects in a quantum dot with a Gaussian confinement. Another important issue to ponder about in this context is whether polaronic interactions can have observable effects in a quantum dot. These effects, if present, deserve to be incorporated in the calculation of the transport and optical properties of a quantum dot because they will be important from the point of view of device applications. It is therefore very important, in this context, to calculate polaronic effects that can be measured experimentally and thus the existence or otherwise of these effects can be substantiated unambiguously. There had been some studies in the past dealing with this aspect but those investigations were either incomplete or were not close enough to real systems [13]. The purpose of the present work is to make a more realistic investigation. In particular, we shall calculate the low-lying electronic energy levels in a GaAs quantum dot in the presence of an external magnetic field and the electron-longitudinal-optical (LO)-phonon interaction for both Gaussian and parabolic potentials incorporating the spin of the electron and study the effect of confinement and polaronic interaction on the Zeeman splitting. Since the Zeeman lines can be experimentally observed, the present study can be decisive in predicting the nature of the confining potential and also the existence or otherwise of polarons in the polar quantum dots.

The Hamiltonian for an electron of Bloch mass $m^{*}$ moving in a two-dimensional (2D) Gaussian potential $V^{\prime}$ and interacting with the LO phonons of dispersionless frequency $\omega_{0}$ of the system in the presence of an external magnetic field $B^{\prime}$ may be written by modifying the Fröhlich Hamiltonian [14,15], as

$$
\begin{aligned}
H^{\prime}= & \frac{\vec{p}^{\prime 2}}{2 m^{*}}+V^{\prime}\left(\vec{\rho}^{\prime}\right)+\frac{1}{8} \omega_{\mathrm{c}}^{\prime 2} \rho^{2}+\frac{\omega_{\mathrm{c}}^{\prime}}{2} L_{z^{\prime}} \\
& +\hbar \omega_{0} \sum_{\vec{q}^{\prime}} b_{\vec{q}^{\prime}}^{\dagger} b_{\vec{q}^{\prime}}+\sum_{\vec{q}^{\prime}}\left(\xi_{\vec{q}^{\prime}}^{\prime} \mathrm{e}^{-\mathrm{i} \vec{q}^{\prime} \cdot \vec{r}^{\prime}} b_{\vec{q}^{\prime}}^{\dagger}+\text { h.c. }\right),
\end{aligned}
$$

where all vectors are $2 \mathrm{D} . \vec{\rho}^{\prime}\left(x^{\prime}, y^{\prime}\right)$ refers to the position vector of the electron, $\vec{p}^{\prime}$ is the corresponding linear momentum operator, $L_{z^{\prime}}$ is the $z$-component of the angular momentum, $\omega_{\mathrm{c}}^{\prime}$ is the bare cyclotron frequency given by $\omega_{c}^{\prime}=e B^{\prime} /\left(m^{*}\right) c, B^{\prime}$ being the magnetic field applied in the $z$-direction, $b_{\vec{q}^{\prime}}^{*}\left(b_{\vec{q}^{\prime}}\right)$ is the creation (annihilation) operator for an LO phonon of wave vector $\vec{q}^{\prime}$ with frequency $\omega_{0}$ and $\xi_{\vec{q}^{\prime}}^{\prime}$ is the electron-phonon interaction coefficient and $V^{\prime}\left(\vec{\rho}^{\prime}\right)$ is the confining potential which we take as

$V^{\prime}\left(\vec{\rho}^{\prime}\right)=-V_{0}^{\prime} \mathrm{e}^{-\rho^{\prime 2} / 2 R^{\prime 2}}$.

We shall work in the Feynman units [16] in which the energy is scaled by $\hbar \omega_{0}$, length by the weak-coupling polaron radius, $r_{0}=\left(\hbar / m^{*} \omega_{0}\right)^{1 / 2}$ and the wave vector by $q_{0}=1 / r_{0}$. This is equivalent to putting $\hbar=m^{*}=\omega_{0}=1$. In these units, the dimensionless Hamiltonian reads

$$
\begin{aligned}
H= & \frac{\vec{p}^{2}}{2}-V_{0} \mathrm{e}^{-\rho^{2} / 2 R^{2}}+\frac{\omega_{\mathrm{c}}}{2} L_{z}+\frac{1}{8} \omega_{\mathrm{c}}^{2} \rho^{2}+\sum_{\vec{q}} b_{\vec{q}}^{\dagger} b_{\vec{q}} \\
& +g_{s} \mu_{\mathrm{B}} B s_{z}+\sum_{\vec{q}}\left(\xi_{\vec{q}} \mathrm{e}^{-\mathrm{i} \vec{q} \cdot \vec{r}} b_{\vec{q}}^{\dagger}+\text { h.c. }\right),
\end{aligned}
$$

where everything is dimensionless; $H=H^{\prime} / \hbar \omega_{0}, \vec{\rho}=\vec{\rho}^{\prime} / r_{0}$, $\vec{q}=\vec{q}^{\prime} / q_{0}, \quad \vec{p}=\vec{p}^{\prime} / \hbar q_{0}, \quad V_{0}=V_{0}^{\prime} / \hbar \omega_{0}, \quad R=R^{\prime} / r_{0}, \quad$ and $\xi_{\vec{q}}=\xi_{\vec{q}}^{\prime} / \hbar \omega_{0}$, where $V_{0}$ is the depth of the confining potential in dimensionless unit, $R$ is the range of the potential giving some kind of a measure of the effective confinement length or the size of the quantum dot and $\xi_{\vec{q}}$ is given by $\left|\xi_{\vec{q}}\right|^{2}=(\sqrt{2} \pi \alpha / v q)$, where $\alpha$ is the dimensionless electron-phonon coupling constant and $v$ represents the volume of the $2 \mathrm{D}$ dot. It may be noted that we have also added in the above Hamiltonian the spin-field interaction where $B$ is the dimensionless magnetic field, $\mu_{\mathrm{B}}$ is the Bohr magneton, $s_{z}$ is the $z$-component of the spin angular momentum of the electron, and $g_{s}$ is the effective Lande- $g$ factor corresponding to the electron spin.

We assume that the deviation of the Gaussian confining potential from the parabolic potential is small enough so that it can be treated as a parabolic potential plus a perturbation. This is a reasonable assumption for small $r$ and since in a quantum dot, $r$ will be generally small, it can be considered as a fairly good approximation. So we rewrite the Hamiltonian (3) as

$H=H_{0}+H_{1}+H_{2}$, 
where

$$
\begin{aligned}
H_{0}= & \frac{\vec{p}^{2}}{2}+\frac{1}{2} \tilde{\omega}^{2} \rho^{2}+\frac{\omega_{\mathrm{c}}}{2} L_{z}+g_{s} \mu_{\mathrm{B}} B s_{z}-V_{0} \\
& +\sum_{\vec{q}} b_{\vec{q}}^{\dagger} b_{\vec{q}},
\end{aligned}
$$

$H_{1}=-\lambda\left[\frac{1}{2} \omega_{\mathrm{h}}^{2} \rho^{2}+V_{0}\left(\mathrm{e}^{-\rho^{2} / 2 R^{2}}-1\right)\right]$,

$H_{2}=\sum_{\vec{q}}\left(\xi_{\vec{q}} \mathrm{e}^{-\mathrm{i} \vec{q} \cdot \vec{r}} b_{\vec{q}}^{\dagger}+\right.$ h.c. $)$,

where $\tilde{\omega}_{\mathrm{h}}^{2}=V_{0} / R^{2}, \tilde{\omega}^{2}=\tilde{\omega}_{\mathrm{h}}^{2}+\omega_{\mathrm{c}}^{2} / 4$ and $\lambda=0$ for a PQD and $\lambda=1$ for a Gaussian quantum dot (GQD). We assume that the sole effect of $H_{1}$ is to renormalize the frequency $\tilde{\omega}$. So we treat $H_{1}$ by the first-order perturbation theory at the mean field level. More specifically we write $H_{1}$ as

$H_{1}=\lambda\left[\frac{V_{0}}{\left\langle\rho^{2}\right\rangle}-\frac{1}{2} \omega_{\mathrm{h}}^{2}-V_{0} \frac{\left\langle\mathrm{e}^{-\rho^{2} / 2 R^{2}}\right\rangle}{\left\langle\rho^{2}\right\rangle}\right] \rho^{2}$,

where $\left\langle\rho^{2}\right\rangle$ is the expectation value of $\rho^{2}$ with respect to ground state wave function of the harmonic oscillator of frequency $\tilde{\omega}$. The GQD problem now essentially reduces to an effective PQD problem described by Hamiltonian

$$
\begin{aligned}
H= & \frac{\vec{p}^{2}}{2}+\frac{1}{2} \omega^{2} \rho^{2}+\frac{\omega_{\mathrm{c}}}{2} L_{z}+g_{s} \mu_{\mathrm{B}} B s_{z}-V_{0} \\
& +\sum_{\vec{q}} b_{\vec{q}}^{\dagger} b_{\vec{q}}+\sum_{\vec{q}}\left(\xi_{\vec{q}} \mathrm{e}^{-\mathrm{i} \vec{q} \cdot \vec{r}} b_{\vec{q}}^{\dagger}+\text { h.c. }\right),
\end{aligned}
$$

where

$$
\begin{aligned}
\omega= & {\left[(1-\lambda) \omega_{\mathrm{h}}^{2}+\frac{\omega_{\mathrm{c}}^{2}}{4}\right.} \\
& \left.+2 \lambda V_{0} \tilde{\omega}\left(1-\left(1+\frac{1}{2 \tilde{\omega} R^{2}}\right)^{-1}\right)\right]^{-1 / 2} .
\end{aligned}
$$

The effective unperturbed problem now satisfies the Schödinger equation

$$
\begin{aligned}
& H_{\mathrm{unp}}\left[\Phi_{n, m}(\vec{\rho}) \chi_{s}(\zeta) \Psi_{\mathrm{ph}}\right] \\
& \quad=\left(E_{n, m, s}^{0}+\varepsilon_{\mathrm{ph}}\right)\left[\Phi_{n, m}(\vec{\rho}) \chi_{s}(\zeta) \Psi_{\mathrm{ph}}\right],
\end{aligned}
$$

where $\Phi_{n, m}(\vec{\rho})$ is the unperturbed electronic wave function given by the Fock-Darwin states

$$
\begin{aligned}
\Phi_{n, m}(\vec{\rho})= & \frac{1}{\sqrt{2 \pi}} \mathrm{e}^{\mathrm{i} m \phi}\left(\frac{2 \omega n !}{n+|m| !}\right)^{1 / 2}(\sqrt{\omega} \rho)^{|m|} \\
& \times L_{n}^{|m|}\left(\omega \rho^{2}\right) \mathrm{e}^{-(1 / 2) \omega \rho^{2}},
\end{aligned}
$$

$\left|\chi_{s}\right\rangle$ is the eigenstate of the operator, $\sigma_{z}=\frac{1}{2} s_{z}$ and corresponds to an up or a down-spin state and $\left|\Psi_{\mathrm{ph}}\right\rangle$ is the eigenstate of the free phonon Hamiltonian $\sum_{\vec{q}} b_{\vec{q}}^{\dagger} b_{\vec{q}}$ and can be written as

$\left|\Psi_{\mathrm{ph}}\right\rangle \equiv|[n]\rangle=\prod_{\vec{q}}\left|n_{\vec{q}}\right\rangle$

with the corresponding energy, $\varepsilon_{\mathrm{ph}}=\sum_{\vec{q}} n_{\vec{q}}$, where $n_{\vec{q}}$ is the number of phonons in the state $\vec{q} . E_{n, m, s}^{0}$ is given by

$$
\begin{aligned}
E_{n, m, s}^{0} & \equiv E_{n, m, \sigma}^{0} \\
& =(2 n+|m|+1) \omega+\left(m-\frac{g_{s} \sigma}{2}\right) \frac{\omega_{\mathrm{c}}}{2}-V_{0},
\end{aligned}
$$

where $\sigma= \pm 1$. The second-order perturbative correction $\Delta E^{(2)}$ due to $H_{2}$ can be written as

$$
\begin{aligned}
& \Delta E_{n m \sigma} \\
& \quad=\sum_{n^{\prime} m^{\prime} \sigma^{\prime}\left[n_{\vec{q}}\right]} \frac{\left|\left\langle\Phi_{n^{\prime} m^{\prime} \sigma^{\prime}}\left|\left\langle\left[n_{\vec{q}}\right]\left|\sum_{\vec{q}}\left(\xi_{\vec{q}} \mathrm{e}^{-\mathrm{i} \vec{q} \cdot \vec{\rho}} b_{\vec{q}}^{\dagger}\right)\right|\left[0_{\vec{q}}\right]\right\rangle\right| \Phi_{n m \sigma}\right\rangle\right|^{2}}{E_{n m \sigma}-E_{n^{\prime} m^{\prime} \sigma^{\prime}}-\sum_{\vec{q}} n_{\vec{q}}} .
\end{aligned}
$$

The summation over the phonon states and $\sigma^{\prime}$ can be performed easily and that over $n^{\prime}, m^{\prime}$ consists in calculating the Green function of the unperturbed problem which can be obtained using the Slater sum rule. We finally obtain for the first few low-lying states

$$
\begin{aligned}
E_{0,0, \pm 1}= & -V_{0} \mp \frac{g_{s} \omega_{\mathrm{c}}}{4} \\
& -\frac{\alpha \sqrt{\pi} \sqrt{\omega}}{2} \int_{0}^{\infty} \frac{\mathrm{e}^{-t}}{\left[1 \pm \mathrm{e}^{-\omega t} \cosh \left(\omega_{\mathrm{c}} t / 2\right)\right]^{1 / 2}} \mathrm{~d} t \\
E_{0,1, \pm 1}=2 & -V_{0}+\left(1 \mp \frac{g_{s}}{2}\right) \frac{\omega_{\mathrm{c}}}{2} \\
& -\frac{\alpha \sqrt{\pi} \sqrt{\omega}}{\sqrt{2}} \int_{0}^{\infty} \frac{\left[2 f(g-h)+h^{2}-f^{2}\right]}{\left[f\left(g f+h^{2}\right)\right]^{3 / 2}} \\
& \times \frac{\mathrm{e}^{-\left(1-\omega-\omega_{\mathrm{c}} / 2\right) t}}{\left(1-\mathrm{e}^{-2 \omega t}\right)} \mathrm{d} t, \\
E_{0,-1, \pm 1}= & 2 \omega-V_{0}+\left(1 \pm \frac{g_{s}}{2}\right) \frac{\omega_{\mathrm{c}}}{2} \\
& -\frac{\alpha \sqrt{\pi} \sqrt{\omega}}{\sqrt{2}} \int_{0}^{\infty} \frac{\left[2 f(g+h)+h^{2}-f^{2}\right]}{\left[f\left(g f+h^{2}\right)\right]^{3 / 2}} \\
& \times \frac{\mathrm{e}^{-\left(1-\omega+\omega_{\mathrm{c}} / 2\right) t}}{\left(1-\mathrm{e}^{-2 \omega t}\right)} \mathrm{d} t .
\end{aligned}
$$

The dipole selection rules allow the following transitions:

$\omega_{\mathrm{c}}^{(+)}=\left[E_{0,1,1}-E_{0,0,1}\right]=\left[E_{0,1,-1}-E_{0,0,-1}\right]$,

$\omega_{\mathrm{c}}^{(-)}=\left[E_{0,-1,-1}-E_{0,0,-1}\right]=\left[E_{0,-1,1}-E_{0,0,1}\right]$.

In the present work, we have considered a GaAs quantum dot but the calculation can be applied to any quantum dot in general. We have of course neglected the spinorbit interaction and as a result we have obtained only two Zeeman lines with the energy splitting equal to $\left(\omega_{\mathrm{c}}^{(+)}-\omega_{\mathrm{c}}^{(-)}\right)$. Inclusion of spin-orbit interaction will obviously cause additional splitting giving rise to a few more Zeeman lines.

In Fig. 1, we show the behaviour of a few low-lying energy levels for a GaAs quantum dot as a function of the range of the potential $R$ for a certain value of $V_{0}$ and the magnetic field. We have plotted results for Gaussian confinement as well as for parabolic confinement for the 


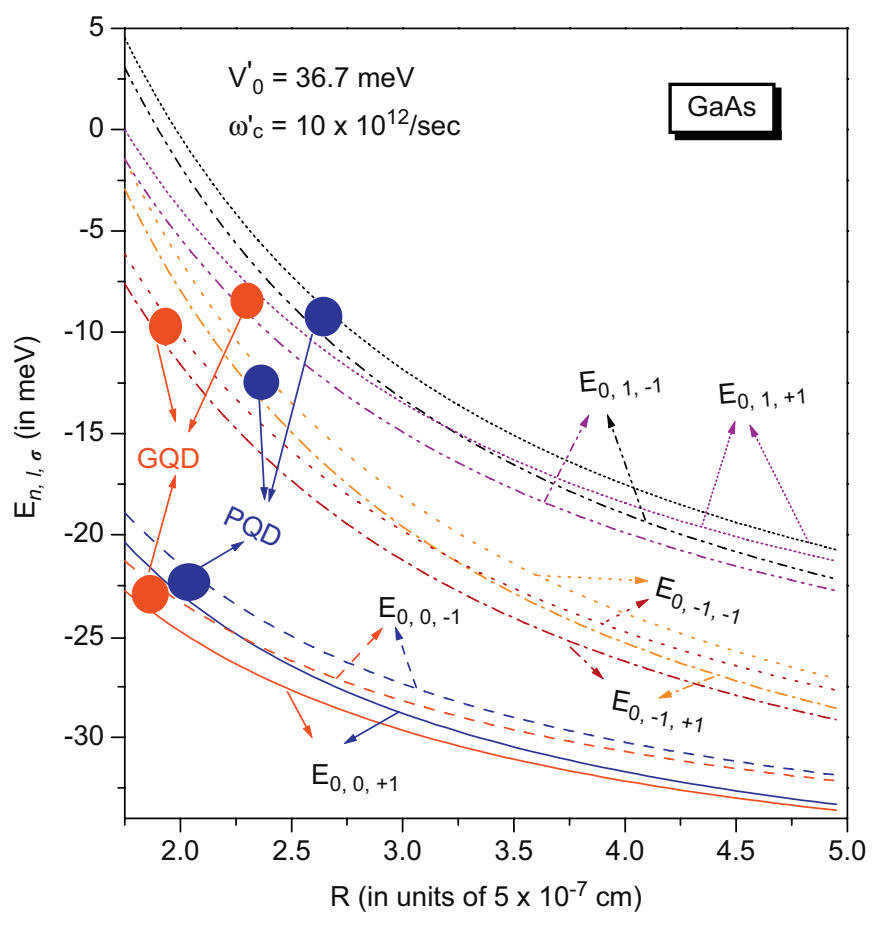

Fig. 1. The total energy of a single-electron GaAs quantum dot in a magnetic field in the presence of electron-phonon interaction as a function of the range of the confining potential, $R$ for $V_{0}^{\prime}=36.7 \mathrm{MeV}$ both for Gaussian and parabolic confining potentials.

sake of comparison. As $R$ decreases, the total energy increases, and this increase becomes more and more rapid for smaller values of $R$. This behaviour arises from the most dominant term of the Gaussian potential which is proportional to $V_{0} / R^{2}$. In the case of the parabolic potential this is of course the only term. As $R$ decreases, this frequency increases rapidly leading to an increase in the total energy. It is evident that the parabolic potential in general gives a higher value for the total energy. This is easy to understand because the additional infinite terms in the Gaussian potential contribute negative energy pushing the total energy down. For large $R$, the difference is, however, rather small between the two potentials, but as $R$ decreases, the gap widens up so much so that a crossing of energy levels occurs at some value of $R$. In Fig. 2, we plot the total energy as a function of the magnetic field again for both parabolic and Gaussian confining potentials for $V_{0}^{\prime}=$ $36.7 \mathrm{meV}$ and $R^{\prime}=12.5 \mathrm{~nm}$. The Zeeman splitting of the energy levels is clearly visible in Fig. 2 . The ground state is split into two levels, one of which increases with the magnetic field while the other one decreases with the magnetic field. The first excited state splits into four levels, two of which increase with the magnetic field while the other two decrease, so that the splitting increases monotonically with the magnetic field. The two levels in the upper branch and as many in the lower one are the spin-split levels. If the spin is ignored one would get only single levels in the upper and lower branches of the excited states while for the ground state there will be no splitting at all. Dipole selection rules, however, allow only two

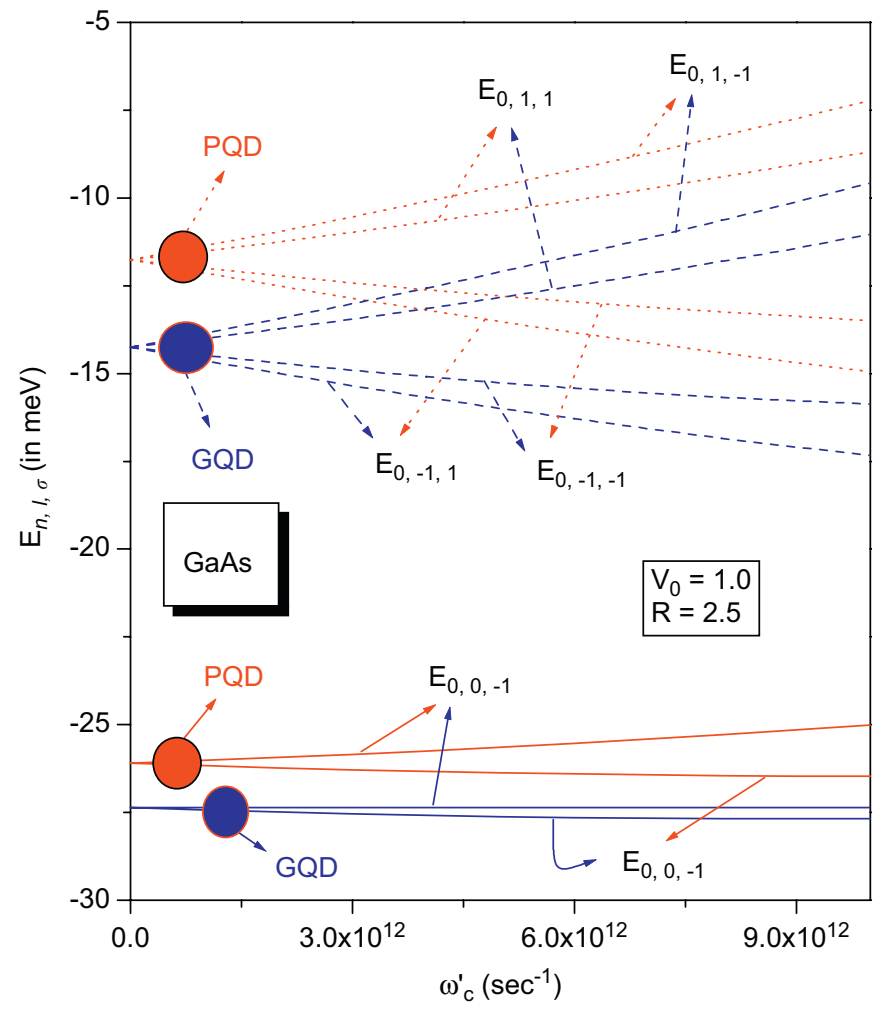

Fig. 2. Zeeman-split energies of the low-lying states as a function of the magnetic field for both Gaussian and parabolic confining potentials for a GaAs quantum dot for $V_{0}^{\prime}=36.7 \mathrm{MeV}$ and $R^{\prime}=12.5 \mathrm{~nm}$.

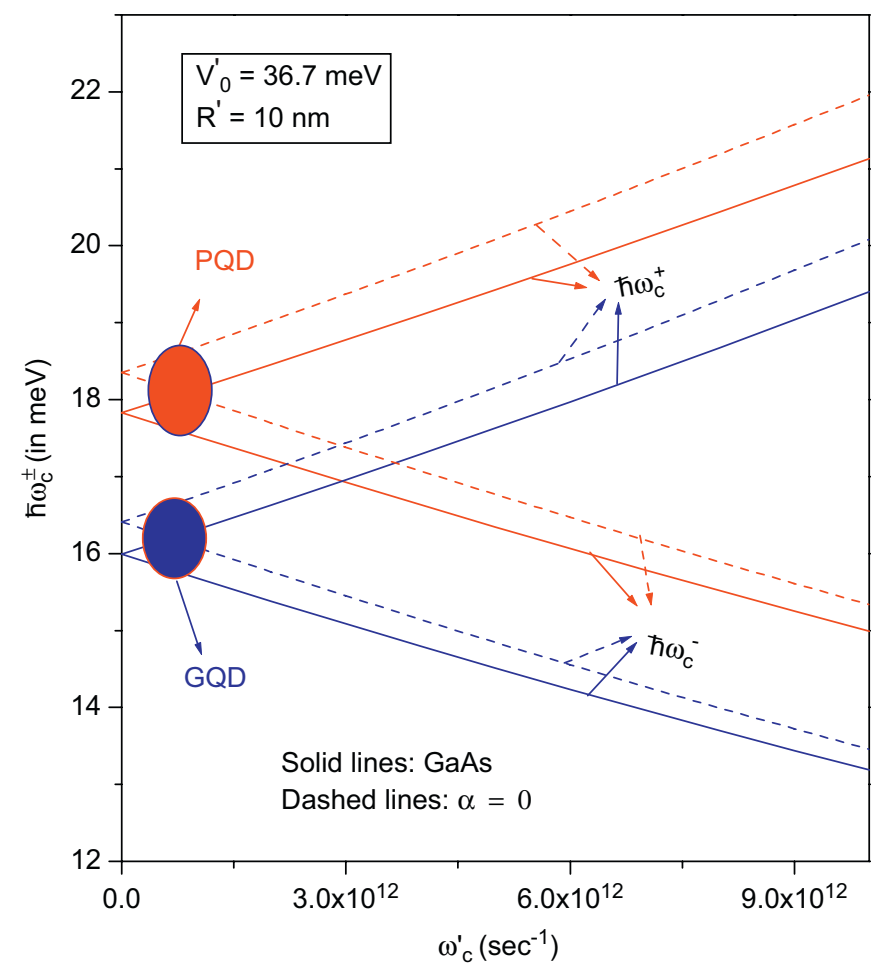

Fig. 3. Cyclotron frequencies, $\hbar \omega_{\mathrm{c}}^{+}$and $\hbar \omega_{\mathrm{c}}^{-}$for a GaAs quantum dot for both Gaussian and parabolic confining potentials for $V_{0}^{\prime}=36.7 \mathrm{MeV}$ and $R^{\prime}=10 \mathrm{~nm}$. The dotted curves show the corresponding results for $\alpha=0$. 
transitions in the present case leading to two cyclotron frequencies, $\omega_{\mathrm{c}}^{+}$and $\omega_{\mathrm{c}}^{-}$. In Fig. 3 we show the variation of these two frequencies for a GaAs quantum dot as a function of the magnetic field for both Gaussian and parabolic confining potentials. We also plot the corresponding curves for $\alpha=0$ for the sake of comparison. One can immediately observe that the parabolic potential overestimates the cyclotron frequencies $\omega_{\mathrm{c}}^{+}$and $\omega_{\mathrm{c}}^{-}$both for $\alpha=0.068$ (GaAs) and $\alpha=0$. For both $\alpha$ values, with increasing magnetic field $\omega_{\mathrm{c}}^{+}$increases while $\omega_{\mathrm{c}}^{-}$decreases. Another important thing is that the cyclotron frequencies turn out to be independent of the spin. This of course will not be true in the presence of the spin-orbit interaction, when the selection rules will be governed by the total angular momentum quantum number, $j$ and the spin $s$ instead of just $m$ and $s$. It is also evident that the inclusion of the electron-phonon interaction reduces the cyclotron frequencies for both parabolic and Gaussian potentials, slightly more for the parabolic potential and furthermore, it also makes the variation of the cyclotron frequencies with the magnetic field a little slower. In quantum dots with higher values of $\alpha$, the effect of electron-phonon interaction is expected to be even larger. All these suggest that electron-phonon interaction cannot be ignored in a polar quantum dot. The reason for the reduction in the cyclotron frequencies in the presence of electron-phonon interaction may be traced as follows. The electron in a quantum dot with an embedded dynamical lattice is neither a bare electron, nor just a Bloch electron, but a complex quasiparticle with a heavier mass because of the formation of a low-energy bound state called a polaron. Thus in the present case, the cyclotron mass is nothing but the polaron mass. Since the effective mass comes in the denominator of the cyclotron frequency, the effective cyclotron frequency is naturally expected to decrease in the presence of the electron-phonon coupling and this is precisely what is happening here. One can also study the variation of the cyclotron mass with $R$. We believe that the values of the cyclotron masses will diminish with increasing effective size of the quantum dot. At this point it may be interesting to look at the polaronic correction to the electron energy. In Fig. 4 we show the variation of the polaron self-energy as a function of $R$ for a GaAs quantum dot for both Gaussian and parabolic potentials. One may note that polaronic effects become strongly pronounced as the size of the quantum dot decreases. As the size increases, the polaronic correction decreases and ultimately saturates to a constant value giving the bulk limit. The polaronic correction, however, as expected, does not depend on the spin state of the electron. We also observe that the parabolic potential overestimates the polaronic effect, more so for smaller values of $R$. Thus we may conclude that parabolic potential is a poor approximation for the Gaussian potential which, as we have already argued, is a more realistic confining potential. As already promised, our major goal is to calculate a quantity that can be measured in the laboratory so that the existence or otherwise of the polaronic effect

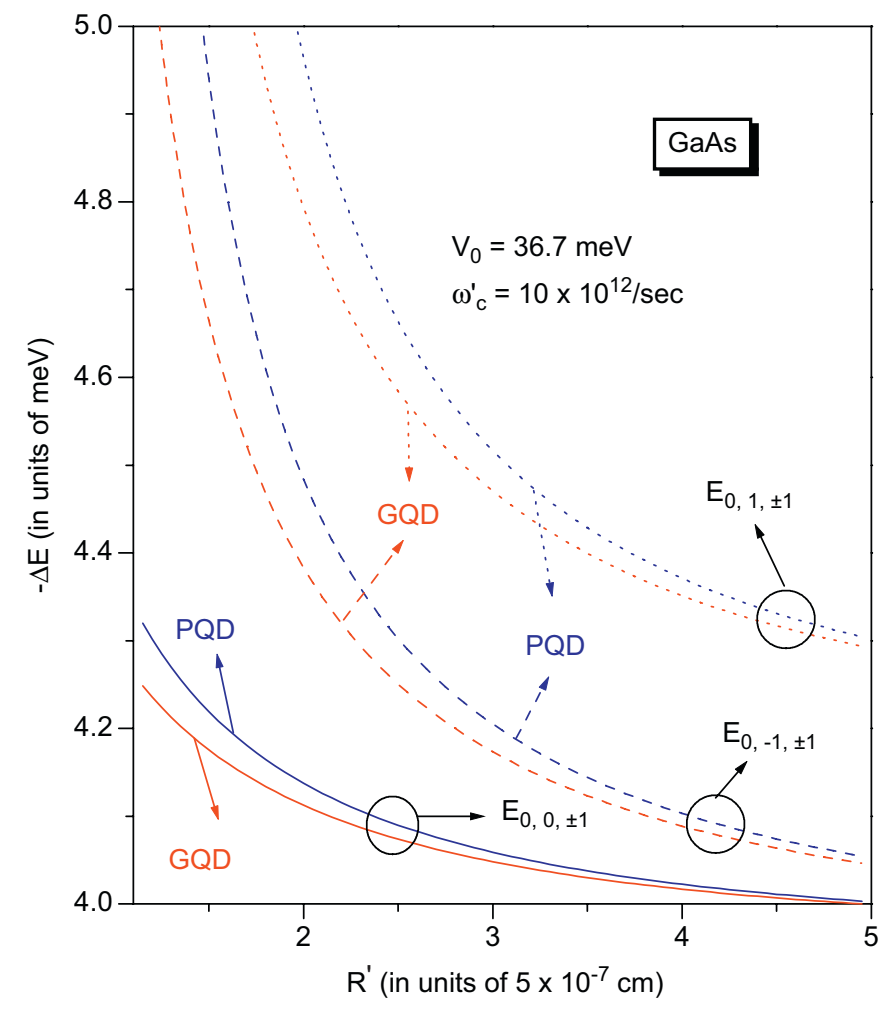

Fig. 4. Polaronic corrections to the electron energy for the first few lowlying states for a GaAs quantum dot for both Gaussian and parabolic confining potentials, for $V_{0}^{\prime}=36.7 \mathrm{MeV}$ and $\omega_{\mathrm{c}}^{\prime}=10 \times 10^{12} / \mathrm{s}$.

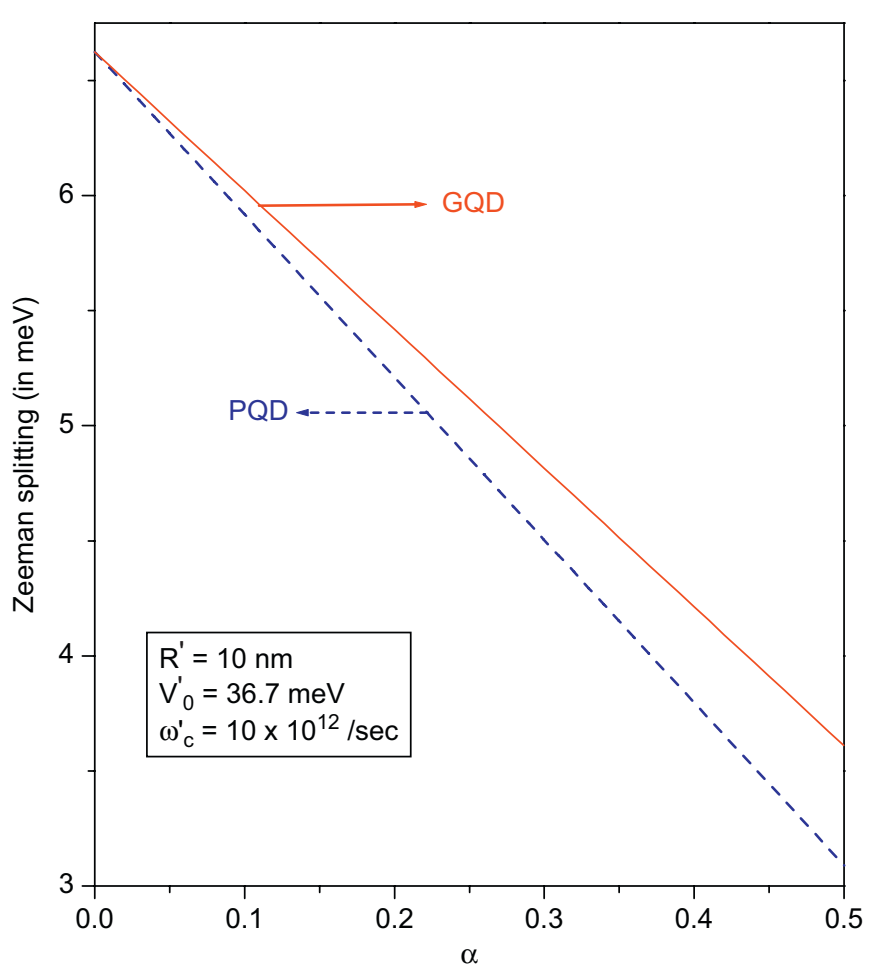

Fig. 5. Zeeman splitting in a GaAs quantum dot for both parabolic and Gaussian confining potentials as a function of $\alpha$ for $R^{\prime}=10 \mathrm{~nm}, V_{0}^{\prime}=$ $36.7 \mathrm{MeV}$ and $\omega_{\mathrm{c}}^{\prime}=10 \times 10^{12}$. 
can be tested experimentally. One such quantity is the Zeeman splitting which is the difference between the two cyclotron frequencies mentioned above. In Fig. 5 we plot the Zeeman splitting as a function of $\alpha$. We find that the Zeeman splitting decreases with the increase of the electron-phonon coupling. This is of course an interesting result. One can of course guess this result from Fig. 3, where we have seen that the cyclotron frequencies are reduced by the electron-phonon coupling and their variations are slower for a GaAs dot than in a dot with $\alpha=0$, other parameters remaining the same. This behaviour can be physically understood in the following way. When an unperturbed excited level of the confining potential in a zero magnetic field lies one LO-phonon energy above the ground level, there is a strong mixing of the excited level with the ground state resulting in devaluation of the magnetic quantum number of the excited level. The magnetic quantum number being responsible for the Zeeman effect, one would then naturally expect a suppression in the Zeeman splitting in the presence of the electron-phonon interaction and this suppression should be more and more as the electro$\mathrm{n}$-phonon coupling is increased. Though this observation is a clear manifestation of the presence of polaronic interaction in a GaAs dot, it would be in a way impossible to realize it in the laboratory since it is not possible to tune the electron-phonon coupling parameter continuously in a particular system. We would therefore like to study the

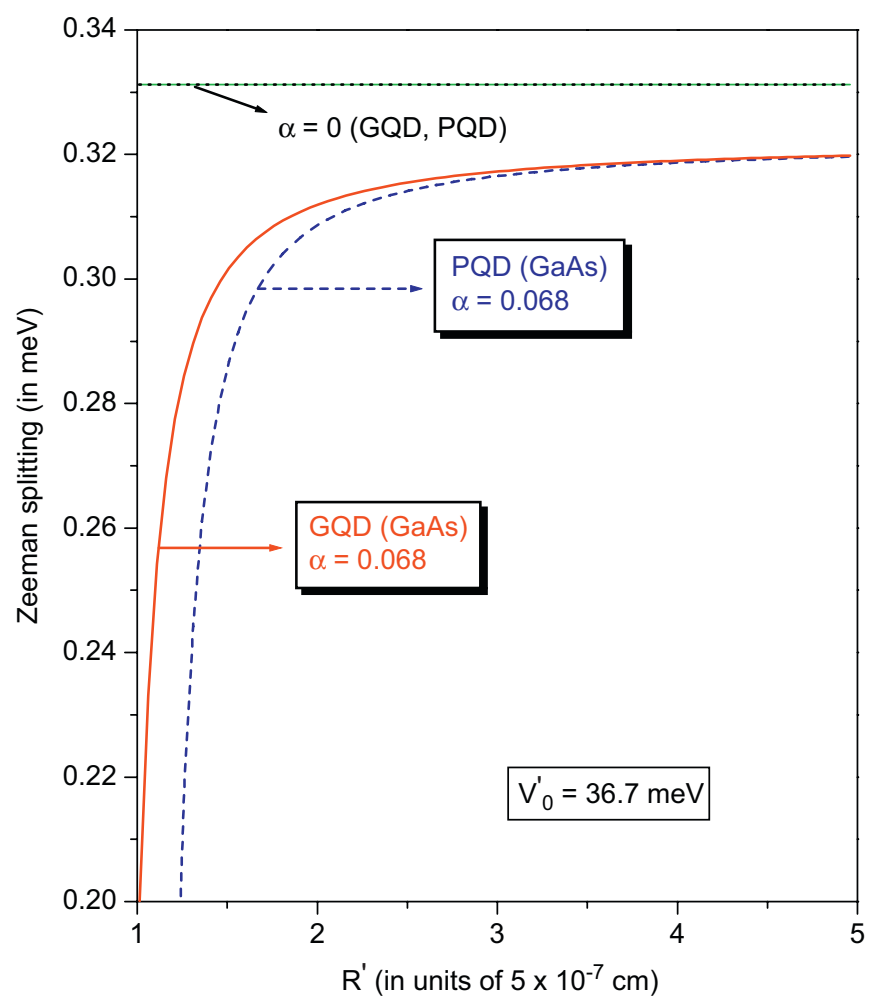

Fig. 6. Zeeman splitting in a GaAs quantum dot as a function of $R$ for both parabolic and Gaussian confining potentials. The case for $\alpha=0$ is also shown for the sake of comparison. variation of the Zeeman splitting as a function of $R$. The results are shown in Fig. 6. One can easily see that in the absence of the electron-phonon interaction, the Zeeman splitting is independent of the size of the quantum dot both for parabolic and the Gaussian potentials. For $\alpha=0.068$, i.e., in $\mathrm{GaAs}$, there is, however, a strong suppression of the Zeeman splitting, particularly as the size of the quantum dot is reduced. The suppression is more in a parabolic dot than in a Gaussian dot. The results are again not difficult to understand. As we have already mentioned, the electron-phonon interaction reduces the Zeeman splitting and this is because of the enhancement in the effective cyclotron mass caused by the polaron formation. With the decrease in the quantum dot size, the polaronic effects become more and more pronounced and as a result the cyclotron mass becomes larger and larger leading to a strongly size-dependent suppression of the Zeeman splitting. Since this effect can be observed experimentally, the existence or otherwise of the polaronic effect in a GaAs quantum dot can be verified experimentally. From the magnitude of the suppression one can also hopefully find out the nature of the confining potential. If observable, this effect can also be utilized to have a desired resonant absorption by tuning the size of the quantum dot using the gate voltage and the lateral confinement.

In conclusion, we have shown that the total energy of a half-spin electron in a GaAs quantum dot in the presence of an external magnetic field and electron-phonon interaction increases with decreasing $R$, because of the concomitant increase in the frequency of the effective oscillator potential. We have also shown that a Gaussian potential generally yields a lower energy than a parabolic potential because it contains infinite additional terms over and above the parabolic potential that altogether makes an overall negative contribution to the total energy. We have furthermore shown that the magnetic field splits the energy levels of the quantum dot which can be termed as a Zeeman effect. In other words, in the presence of a confining potential and the electron-phonon interaction, the energy levels of a spin-half electron can be considered as modified Landau levels. we find that for low-energy processes, the dipole selection rules allow only two distinct transitions between these Landau levels giving rise to two cyclotron frequencies one of which increases with the magnetic field while the other one decreases. It is also observed that inclusion of spin does not affect the cyclotron frequencies at all, though it does influence the splitting of the individual energy levels. However, even in the present case, the effect of the spin is expected to show up in the thermodynamic quantities which require the actual energy spectrum for their calculation. Of course, the situation will certainly change if the spin-orbit coupling is introduced. It turns out that a parabolic confining potential overestimates the cyclotron energies. But the more important observation, in our opinion, is the reduction in the cyclotron frequencies due to the electron-phonon interaction. This result can be attributed to the polaronic 
effect. Because of the polaron formation in a GaAs quantum dot, the effective cyclotron mass undergoes an enhancement leading to a devaluation of the cyclotron frequencies. The polaron self-energies for the first few lowlying states suggest that the polaronic effects become quite significant if the quantum dot size is reduced below a few nanometers. We have observed that the Zeeman splitting decreases almost linearly with the increasing value of the phonon coupling and the parabolic potential overestimates this suppression as compared to a Gaussian potential. Finally we have shown that the suppression of the Zeeman splitting is strongly size dependent below a certain size of the quantum dot and here again this suppression is found to be overestimated by the parabolic potential. Thus, we may conclude that a parabolic potential leads to sizable deviations in the results vis-a-vis a Gaussian potential and so it would be more practical to use the Gaussian potential as a confining potential instead of a parabolic potential for theoretical calculations. We claim that the size-dependence of the Zeeman suppression should be observable in the laboratory through infrared magneto-optical experiments and would give an unambiguous experimental evidence of the existence or otherwise of the polaronic effects in a GaAs quantum dot. The size-dependent Zeeman suppression is also a clear manifestation of the quantum size effect and can be usefully exploited to obtain a desired Zeeman splitting just by tuning the confining potentials and the gate voltage to achieve resonant absorption. This effect can also be useful in quantum dot lasers.

\section{References}

[1] M.A. Kastner, Rev. Mod. Phys. 64 (1992) 849; N.F. Johnson, J. Phys.: Condens. Matter 7 (1995) 965; R.C. Ashoori, Nature 379 (1996) 413;

U. Woggon, Optical Properties of Semiconductor Quantum Dots, Springer, Berlin, 1997;

L. Jack, P. Hawrylack, A. Wojs, Quantum Dots, Springer, Berlin, 1998;

D. Bimberg, M. Grundmann, N.N. Ledentsov, Quantum Dot Heterostructures, Wiley, New York, 1998;

S. Mukhopadhyay, A. Chatterjee, Acta Phys. Pol. B 32 (2001) 473.

[2] Ch. Sikorski, U. Merkt, Phys. Rev. Lett. 62 (1989) 2164;

Ch. Sikorski, U. Merkt, Surf. Sci. 229 (1990) 282;

B. Meurer, D. Heitmann, K. Ploog, Phys. Rev. Lett. 68 (1992) 1371; R.C. Ashoori, H.L. Stormer, J.S. Weiner, L.N. Pfeiffer, S.J. Pearton, K.W. Balswin, K.W. West, Phys. Rev. Lett. 68 (1992) 3088;

H. Drexler, D. Leonard, W. Hansen, J.P. Kotthaus, P.M. Petroff, Phys. Rev. Lett. 73 (1994) 2252.

[3] F.M. Peeters, Phys. Rev. B 42 (1990) 1486;

S.K. Yip, Phys. Rev. B 43 (1991) 1707;
Q.P. Li, K. Karrai, S.K. Yip, S. Das Sarma, H.D. Drew, Phys. Rev. B 43 (1991) 5151.

[4] W. Kohn, Phys. Rev. 123 (1961) 1242.

[5] P.A. Maxym, T. Chakraborty, Phys. Rev. Lett. 65 (1990) 108; N.F. Johnson, M.C. Payne, Phys. Rev. Lett. 67 (1991) 1157; N.F. Johnson, F.C. Payne, Phys. Rev. B 45 (1992) 3819;

R. Egger, W. Hausler, C.H. Mak, H. Grabert, Phys. Rev. Lett. 82 (1999) 3320.

[6] S.W. Gu, K.X. Guo, Solid State Commun. 89 (1993) 1023; K.D. Zhu, S.W. Gu, Phys. Rev. B 47 (1993) 12947;

K.D. Zhu, T. Kobayashi, Solid State Commun. 95 (1995) 805;

C.Y. Chen, P.W. Jin, W.S. Li, D.L. Lin, Phys. Rev. 56 (1997) 14913;

S. Mukhopadhyay, A. Chatterjee, Phys. Lett. A 242 (1998) 355;

S. Mukhopadhyay, A. Chatterjee, Phys. Lett. A 204 (1995) 411;

S. Mukhopadhyay, A. Chatterjee, Int. J. Mod. Phys. 10 (1996) 2781;

S. Mukhopadhyay, A. Chatterjee, Phys. Rev. B 55 (1997) 9297;

S. Mukhopadhyay, A. Chatterjee, Phys. Rev. B 58 (1998) 2088;

S. Mukhopadhyay, A. Chatterjee, Phys. Lett. A 240 (1998) 100;

S. Mukhopadhyay, A. Chatterjee, Phys. Rev. B 59 (1999) R7833;

S. Mukhopadhyay, A. Chatterjee, J. Phys.: Condens. Matter 11 (1999) 2071;

S. Mukhopadhyay, A. Chatterjee, Int. J. Mod. Phys. B 14 (2000) 3897;

S. Mukhopadhyay, A. Chatterjee, Int. J. Mod. Phys. B 16 (2002) 1489 ;

N. Kervan, T. Altanhan, A. Chatterjee, Phys. Lett. A 315 (2003) 280; P.M. Krishna, A. Chatterjee, Physica B 358 (2005) 191;

P.M. Krishna, A. Chatterjee, Physica E 30 (2005) 64;

P.M. Krishna, S. Mukhopadhyay, A. Chatterjee, Solid State Commun. 138 (2006) 285;

P.M. Krishna, S. Mukhopadhyay, A. Chatterjee, Phys. Lett. A 360 (2007) 655.

[7] D. Heitmann, K.K. Bollweg, V. Gudmundsson, T. Kurth, S.P. Riege, Physica E 1 (1997) 204;

B.T. Miller, W. Hansen, S. Manus, R.J. Luyken, A. Lorke, J.P. Kotthaus, S. Huant, G. Mediros-Ribeiro, P.M. Petroff, Phys. Rev. B 56 (1997) 6764.

[8] B. Szfran, J. Adamowsky, S. Bednarek, Physica E 4 (1999) 1.

[9] S. Bednarek, B. Szafran, J. Adamowsky, Phys. Rev. B 59 (1999) 13036.

[10] B. Szfran, J. Adamowsky, S. Bednarek, Phys. Rev. B 61 (2000) 1971.

[11] J. Adamowsky, M. Sobkowicz, B. Szafran, S. Bednarek, Phys. Rev. B 62 (2000) 4234.

[12] N. Bessis, H. Friedrich, C. Wheatly, J. Phys. A 15 (1982) 3679; C.S. Lai, J. Phys. A 16 (1983) L181;

R.E. Crandall, J. Phys. A 16 (1983) L395;

M. Cohen, J. Phys. A 17 (1984) L101;

A. Chatterjee, J. Phys. A 18 (1985) 2403;

A. Chatterjee, Phys. Rep. 186 (1990) 249.

[13] S. Mukhopadhyay, A. Chatterjee, Phys. Rev. B 59 (1999) R7833; P.M. Krishna, S. Mukhopadhyay, A. Chatterjee, Int. J. Mod. Phys. B 16 (2002) 1489.

[14] T.K. Mitra, A. Chatterjee, S. Mukhopadhyay, Phys. Rep. 153 (1987) 91.

[15] H. Fröhlich, Philos. Mag. Suppl. 3 (1954) 325

[16] R.P. Feynman, Phys. Rev. 97 (1955) 660. 